\title{
Interpretation of doxycycline+chloroquine dual therapy for $A$. phagocytophilum infection in dogs
}

\author{
Interpretación de terapia dual de doxicilina+cloroquina para la \\ infección con A. phagocytophilum en perros
}

\begin{abstract}
Kerem Ural, Ph.D.
Adnan Menderes University, Faculty of Veterinary, Department of Internal Medicine, Isikli, Aydin, Turkey. *Corresponding: uralkerem@gmail.com
\end{abstract}

Received: March 2014; Accepted: January 2015.

\begin{abstract}
Objective. A. phagocytophilum, an obligate intracellular pathogen, is a well-known agent causing granulocytic infections in both animals and humans. The purpose of the present study was to describe clinical course and consequences of Canine Granulocyctic Anaplasmosis among dogs in Aydin province, Turkey with special reference to hematological alterations and possible interpretations of doxycycline+chloroquine dual therapy. Materials and methods. A controlled clinical trial was carried out on 14 dogs referred and diagnosed as Canine Granulocyctic Anaplasmosis within Snap 4dx test. Relevant haematological data were recorded before (day 0) and after treatment (day 30) in both groups. Group I ( $n=7)$ were adminestered doxycycline (10 mg/kg q $12 \mathrm{~h}$ via oral route for 14 days) and chloroquine $(2.5 \mathrm{mg} / \mathrm{kg} \mathrm{q} 12 \mathrm{~h}$ for 14 days) and group II $(\mathrm{n}=7)$ received only doxycycline (10 $\mathrm{mg} / \mathrm{kg} \mathrm{q} 12 \mathrm{~h}$ for 14 days via oral route) therapy. Results. Doxycycline treatment hasten resolution of clinical signs in all dogs in about 2 to 7 days. There was no statistically significant differences among hematological variances detected $(p>0.05)$. Conclusions. It may suggest that in conjunction with doxycycline chloroquine may have helped to speed up relevant clinical signs of CGA.
\end{abstract}

Key words: Anaplasmosis, Canine, chloroquine, doxycycline, granulocyctic (Source: $C A B$ ).

\section{RESUMEN}

Objetivo. A. phagocytophilum, un patógeno intracelular obligado, es un agente ampliamente conocido que causa infecciones granulocíticas tanto en animales como en humanos. El propósito del presente estudio fue describir la evolución clínica y las consecuencias de la Anaplasmosis Granulocítica Canina en perros de la provincia de Aydin, Turquía, con especial referencia a las alteraciones hematológicas y a las posibles interpretaciones de una terapia dual de doxicilina+cloroquina. Materiales y métodos. Se realizó un estudio clínico controlado en 14 perros remitidos y diagnosticados con Anaplasmosis Granulocítica Canina usando de una prueba Snap 4dx. Se registraron datos hematológicos pertinentes antes (día 0) y después del tratamiento (día 30) en ambos grupos. Al Grupo I $(n=7)$ se le administró doxicilina (10 mg/kg q $12 \mathrm{~h}$ por vía oral durante 14 días) y cloroquina ( $2.5 \mathrm{mg} / \mathrm{kg} \mathrm{q} 12 \mathrm{~h}$ durante 14 días), mientras que el Grupo II ( $n=7)$ recibió una terapia únicamente con doxicilina $(10 \mathrm{mg} / \mathrm{kg} \mathrm{q} 12$ h por vía oral durante 14 días). Resultados. La doxicilina aceleró la resolución de los signos clínicos en todos los perros en un periodo de aproximadamente 2 a 7 días. No se detectaron diferencias estadísticas significativas entre las variaciones hematológicas $(p>0.05)$. Conclusiones. Lo anterior puede sugerir que, conjuntamente con la doxicilina, la cloroquina puede haber ayudado a acelerar los signos clínicos pertinentes de la Anaplasmosis Granulocítica Canina (AGC).

Palabras clave: Anaplasmosis, canino, doxycycline, chloroquine, granulocyctico (Fuente: CAB). 
Ural - Doxycycline chloroquine combination therapy of Anaplasmosis in dogs

\section{INTRODUCTION}

The canine vector-borne infectious diseases are emerging problems in veterinary medicine, besides the zoonotic potential of the latter causative agents may carry of importance for human health (1). The vector-borne canine diseases, especially human granulocytic ehrlichiosis caused by Anaplasma phagocytophilum (A. phagocytophilum) is frequently observed worldwide. Ticks are well recognized as the main method of transmission of the HE.

Anaplasma phagocytophilum is a well recognized agent of granulocytic anaplasmosis, possessing influence on neutrophils and rarely eosinophils (2). A. phagocytophilum infections can be detected either directly in blood smears (morulae in granulocytes) or by PCR or indirectly by serology. Many laboratories perform serological testing for IgG antibodies by using indirect immunofluorescent antibody techniques (IFAT).

Regarding human granulocytic anaplasmosis, diagnosis rely upon relevant clinical signs and laboratory analysis as follows; (i) microscobic morulae observation among neutrophils on satined blood smears along with antibody titer positivity against $A$. phagocytophilum; (ii) a 4-fold antibody titer elevation/reducing in 1 month; (iii) PCR positivity; or (iv) $A$. phagocytophilum identification and isolation in blood sample (3). Aforementioned criteria may also be adapted to dogs, apart from microbiological isolation $(2,4-6)$.

Doxycycline is the armoured therapy option for Canine Granulocyctic Anaplasmosis (CGA). Indeed further research are warranted in an attempt to investigate any adjuvant or combined therapy alternatives for better releiving the hematopathogical and clinical signs. The aim of this study was to analyze clinical consequences of CGA among dogs, besides to scrutinize hematological alterations and interpretation of doxycycline+ chloroquine dual therapy.

\section{MATERIAL AND METHODS}

Conclusion criteria, sampling. The present study was enrolled among 14 dogs referred to the Small Animal Clinics at the Department of Internal Medicine, Faculty of Veterinary, Adnan Menderes University and privately owned small animal clinics in Aydin province between 2013 March-2014 February. All diseased dogs, referred with histories of at least one of the

\section{INTRODUCCIÓN}

Las enfermedades caninas infecciosas trasmitidas por vectores representan un problema emergente en la medicina veterinaria; además, el potencial zoonótico de los agentes causales puede ser de importancia para la salud humana (1). Las enfermedades caninas transmitidas por vectores, especialmente la ehrlichiosis granulocítica humana causada por Anaplasma phagocytophilum ( $A$. phagocytophilum), se observan con frecuencia a nivel mundial. Las garrapatas han sido ampliamente reconocidas como el principal medio de transmisión de la EH.

Anaplasma phagocitophylum es un agente ampliamente reconocido de la anaplasmosis granulocítica, que ejerce una influencia sobre los neutrófilos y raras veces sobre los eosinófilos (2). Las infecciones de a+ se pueden detectar ya sea directamente en frotis de sangre (mórulas en granulocitos), por reacción en cadena de la polimerasa o indirectamente por serología. Varios laboratorios realizan pruebas serológicas de anticuerpos IgG técnicas indirectas de anticuerpos inmunofluorescentes (IFAT por su sigla en inglés).

En cuanto a la anaplasmosis granulocítica humana, los diagnósticos dependen de signos clínicos pertinentes y análisis de laboratorio de la siguiente manera; (i) observación de mórulas microscópicas en neutrófilos en frotis de sangre junto con positividad de título de anticuerpos contra a+; (ii) una elevación/reducción cuádruple de título de anticuerpo en 1 mes; (iii) positividad de reacción en cadena de polimerasa; o (iv) identificación y aislamiento en sangre de a+ (3). Los criterios antes mencionados también se pueden adaptar a perros, salvo por el aislamiento microbiológico $(2,4-6)$.

La doxicilina es la opción terapéutica blindada para la Anaplasmosis Granulocítica Canina (AGC). En efecto, se justifica realizar investigaciones adicionales para investigar alternativas terapéuticas adyuvantes o combinadas para mejorar el alivio de signos hematopatológicos y clínicos. El objetivo de este estudio fue analizar las consecuencias clínicas de la AGC en perros, como también mirar en detalle las alteraciones hematológicas e interpretar de la terapia dual con doxicilina+ cloroquina.

\section{MATERIALES Y MÉTODOS}

Criterios de conclusión, muestreo. El presente estudio se llevó a cabo entre 14 perros remitidos a las Clínicas de Animales Pequeños del Departamento de Medicina Interna, Facultad de Veterinaria, Universidad Adnan Menders y en pequeñas clínicas privadas en la provincia de Aydin entre marzo de 2013 y febrero de 2014. Todos los perros 
clinical signs involving fever, anorexia, weight loss, fever, generalized lymphadenopathy, arthropathy, muscle weakness, epistaxis and distal limb edema were evaluated using a canine point-of-care ELISA kit for diagnosis of naturally occuring vector borne diseases (SNAP 4DX, IDEXX Laboratories, USA). Informed written consent was obtained from all of the dogs owners prior to enrolment of the dogs participated in study $(n=14$, at the age of 16 months to 4 years, 8 male, 6 female).

\section{Laboratory analyses}

Haematological analysis. Blood samples were withdrawn from vena cephalica antebrachii into anticoagulated (EDTA) tubes. Complete blood counts were performed on referral within Abacus Junior Vet hematology analyzer.

Serological analysis. Sera samples were tested within ELISA kit (SNAP 4Dx, IDEXX Laboratories, USA) in an attempt to diagnose antibodies against $A$. phagocytophilum, Ehrlichia canis, and Borrellia burgdorferi and antigen of Dirofilaria immitis, according to the protocol listed in the product insert. This assay detects antibodies reacting to immunodominant protein (msp2) of A. phagocytophilum. Only dogs naturally and mono-infected with $A$. phagocytophilum were enrolled in the present study. The test results were recorded in an Excel spreadsheet.

Treatment procedure. Fourteen dogs with a diagnosis of CGA were randomly enrolled into 2 groups. Treatment in both groups involved peroral administration. Group I $(n=7)$ received doxycycline [(Monodoks capsule, $100 \mathrm{mg}$, Deva, Turkey) (10 mg/kg q $12 \mathrm{~h}$ for 14 days)] + chloroquine [(Kutlu tablet, 250 mg, Abdi İbrahim, Turkey) (2.5 mg/kg $12 \mathrm{~h}$ for 14 days)] and group II $(n=7)$ received mono-doxycycline $(10 \mathrm{mg} / \mathrm{kg}$ $\mathrm{q} 12 \mathrm{~h}$ for 14 days) therapy.

Statistical analysis. Data were analyzed using the Mann-Whitney $U$ (inter-group comparison) and the Wilcoxon test (intra-group comparison) procedures.

\section{RESULTS}

Serological results. Sera samples were tested within ELISA kit (SNAP 4Dx, IDEXX Laboratories, USA) revealed antibodies reacting to immunodominant protein ( $\mathrm{msp} 2$ ) of $A$. phagocytophilum in all cases.

Clinical cure. Treatment in both groups resulted in rapid resolution of clinical signs in all dogs in about 2 to 7 days. Although not significant and statistically not important (data enfermos, remitidos con al menos un signo clínico que involucrara fiebre, anorexia, pérdida de peso, linfadenopatía, artropatía, debilidad muscular, epistaxis y edema distal en miembros, fueron evaluados utilizando un kit de ELISA canino en el punto de atención para diagnosticar enfermedades transmitidas por vectores que ocurren naturalmente (SNAP 4DX, IDEXX Laboratories, EE.UU.). Se obtuvo consentimiento informado por escrito por parte de todos los dueños de los perros que participaron antes de vincularlos al estudio $(n=14$, en edades desde los 16 meses hasta los 4 años, 8 machos, 6 hembras).

\section{Análisis de laboratorio}

Análisis hematológico. Se tomaron muestras de sangre de la vena cephalica antebrachii en tubos anticoagulados (ácido etildiaminotetraacético EDTA).Se realizaron conteos de sangre completos al ser remitidos utilizando un analizador de hematología Abacus Junior Vet.

Análisis serológico. Se tomaron muestras de suero utilizando un kit de ELISA (SNAP 4Dx, IDEXX Laboratories, EE.UU.) buscando diagnosticar los anticuerpos contra A. phagocytophilum, Ehrlichia canis, y Borrellia burgdorferiy y el antígeno de Dirofilaria immitis, de acuerdo al protocolo relacionado en el folleto del producto. Este ensayo detecta anticuerpos que reaccionan a las proteínas inmunodominantes (msp2) de A. phagocytophilum. En este estudio participaron sólo participaron perros infectados únicamente con $A$. phagocytophilum. Los resultados de las pruebas se registraron en una hoja de cálculo de Excel.

Procedimiento del tratamiento. Catorce perros diagnosticados con AGC fueron inscritos aleatoriamente en 2 grupos. En ambos grupos, el tratamiento involucró una administración peroral. El grupo I $(n=7)$ recibió una terapia de doxicilina [(Monodokus cápsula, 100 mg, Deva, Turquía) (10 mg/kg q $12 \mathrm{~h}$ durante 14 días) ] + cloroquina [(Kutlu tableta, $250 \mathrm{mg}$, Abdi İbrahim, Turquía) ( $2.5 \mathrm{mg} / \mathrm{kg} 12 \mathrm{~h}$ durante 14 días)] y el grupo II $(n=7)$ recibió una terapia únicamente con doxicicilina ( $10 \mathrm{mg} / \mathrm{kg}$ q $12 \mathrm{~h}$ durante 14 días).

Análisis estadístico. Los datos se analizaron utilizando como procedimientos la U Mann-Whitney (comparación intergrupal) y la prueba de Wilcoxon (comparación intragrupal).

\section{RESULTADOS}

Resultados serológicos. Las pruebas de las muestras de suero se llevaron a cabo en un kit de ELISA (SNAP 4Dx, IDEXX Laboratories, EE.UU.), revelando los anticuerpos que reaccionaron 
not shown) dogs enrolled in group I, that were dual treated, presented a more rapid clinical cure. Treatment in both groups resulted with clinical remission regarding aforementioned clinical signs. On day 30 no fever was evident in treated dogs. Spontaneous and gradual regression of enlarged lymp nodes occurred in 4 to 12 days in both groups. Finnaly on day 30 all dogs presented complete clinical recovery.

Analysis for relevant hematological variables. No significant difference was found among the mean hematological values of the two groups before and after treatment ( $p>0.05)$ (Table 1). Intra-group comparison revealed that there was no significant difference between mean values of hematological variances before and after treatment $(p>0.05)$. Solely in doxycycline group showed significantly altered mean values regarding $\mathrm{RBC}, \mathrm{Hb}, \mathrm{HCT}$ and PLT before and after treatment $(p \leq 0.05)$.

\section{DISCUSSION}

A. phagocytophilum, capable of infecting granulocytes, and so far neutrophils and eosinophils (7), frequently cause an acute febrile systemic illness, namely CGA. In an attempt to make precise diagnostic tests for CGA microscopic detection of specific morulae, anti-Anaplasma IgM and IgG antibody detection and PCR analysis, which is most reliable for early diagnosis (8).

Recent exposure to $A$. phagocytophilum may be determined in dogs and human by acute and convalescent serologic testing, to those of IFA assays (9). IgG class antibodies primarily are detectable following 8 days after first exposure, corresponding to 2-5 days after morulae presence. It is therefore may be suggested that antibody detection may not be possible during acute infection (7). Seronegative dogs could probably have been recently infected; indeed a seropositive case could have been exposed to the etiological agent preceeding months, in association with elimination of infection thorugh immunity (10). Polymerase chain reaction ( $P C R$ ) assays can be used to detect organism-specific DNA sequences in the blood during the early stages (11). Therefore, the present authors may suggest that seropositive dogs obtained in the present study may have been infected weeks to months earlier, similar to previous description (9).

The major flaw in the present research was that serological or molecular testing results at the time of diagnosis was not available, a la proteína inmunodominante $(\mathrm{msp} 2)$ de $A$. phagocytophilum en todos los casos.

Curación clínica. El tratamiento en ambos grupos dio como resultado una rápida resolución de los signos clínicos en todos los perros en un periodo de aproximadamente 2-7 días. Los perros en el grupo I, que recibieron tratamiento dual, presentaron una curación clínica más rápida, aunque no significativamente y sin importancia estadística (no se muestran los datos). El tratamiento en ambos grupos resultó en la remisión clínica respecto de los signos clínicos antes mencionados. En el día 30 no se observó una presencia evidente de fiebre en los perros sometidos a tratamiento. En ambos grupos se observó una regresión espontánea y gradual de los ganglios linfáticos entre los 4 y los 12 días. Finalmente, en el día 30 todos los perros presentaron una recuperación clínica total.

Análisis de las variables hematológicas pertinentes. No se encontró una diferencia significativa antes y después del tratamiento entre los valores hematológicos medios de los dos grupos ( $p>0.05)$ (Tabla 1). La comparación intragrupal reveló que no hubo una diferencia significativa entre los valores medios de las varianzas hematológicas antes y después del tratamiento $(p>0.05)$. únicamente el grupo tratado con doxicilina mostró valores medios alterados significativamente en cuanto a RBC, Hb, HCT y PLT antes y después del tratamiento $(p \leq 0.05)$.

\section{DISCUSIÓN}

A. Phagocytophilum, capaz de infectar a los granulocitos, y a saber también a los neutrófilos y a los eosinófilos (7), a menudo causa una enfermedad febril aguda, concretamente AGC. En un intento por hacer precisas las pruebas de diagnóstico de detección microscópica de mórulas en AGC, se utilizó la detección de anticuerpos IgM e IgC antiAnaplasma y el análisis de reacción en cadena de la polimerasa, ya que son los más fiables para un diagnóstico temprano (8).

Es posible determinar una exposición reciente a $A$. phagocytophilum en perros y en humanos por medio de pruebas de sueros agudos $y$ convalecientes, con ensayos indirectos de anticuerpos inmunofluorescentes (IFA) (9).

Los anticuerpos de clase IgG son detectables mayormente a los 8 días de la primera exposición, correspondientes a 2-5 días posteriores a la presencia de mórulas. Es por ello que se puede sugerir que no es posible la detección de anticuerpos durante la infección aguda (7). Es probable que los perros seronegativos hayan sido infectados recientemente; en efecto, un caso seropositivo 
Table 1. Hematological variances and relevant statistical analysis.

\begin{tabular}{|c|c|c|c|c|}
\hline \multirow{2}{*}{ Parameters } & \multicolumn{2}{|c|}{ Group I } & \multicolumn{2}{|c|}{ Group II } \\
\hline & Before & After & Before & After \\
\hline WBC $\left(\times 10^{9} / I\right)$ & $\begin{array}{l}15.5 \pm 2.20 \\
(6.7-22.1)\end{array}$ & $\begin{array}{l}11.5 \pm 1.24 \\
(7.6-15.9)\end{array}$ & $\begin{array}{l}15.5 \pm 2.44 \\
(6.2-25.3)\end{array}$ & $\begin{array}{l}11.2 \pm 1.31 \\
(6.7-16.0)\end{array}$ \\
\hline $\operatorname{RBC}\left(\times 10^{12} / \mathrm{I}\right)$ & $\begin{array}{l}5.6 \pm 0.29 \\
(4.0-6.4)\end{array}$ & $\begin{array}{l}6.8 \pm 0.49 \\
(5.0-8.1)\end{array}$ & $\begin{array}{l}5.0 \pm 0.44 \\
(4.0-6.6)\end{array}$ & $\begin{array}{l}6.3 \pm 0.39 \\
(4.6-7.5)\end{array}$ \\
\hline HG (g/dl) & $\begin{array}{l}12.5 \pm 0.74 \\
(8.5-14.3)\end{array}$ & $\begin{array}{c}14.8 \pm 1.32 \\
(10.0-18.0)\end{array}$ & $\begin{array}{l}11.0 \pm 1.19 \\
(7.9-15.0)\end{array}$ & $\begin{array}{c}14.4 \pm 0.76 \\
(11.3-16.8)\end{array}$ \\
\hline HCT (\%) & $\begin{array}{c}37.2 \pm 2.06 \\
(25.6-41.1)\end{array}$ & $\begin{array}{c}44.6 \pm 3.16 \\
(31.5-51.7)\end{array}$ & $\begin{array}{c}32.8 \pm 3.13 \\
(25.4-43.2)\end{array}$ & $\begin{array}{l}42.2 \pm 2.36 \\
(33.6-50.7)\end{array}$ \\
\hline PLT (x10/I) & $\begin{array}{c}155.4 \pm 71.58 \\
(9.0-557)\end{array}$ & $\begin{array}{c}336.0 \pm 57.75 \\
(224-619)\end{array}$ & $\begin{array}{c}107.1 \pm 33.01 \\
(5.0-248)\end{array}$ & $\begin{array}{c}461.5 \pm 42.65 \\
(328-619)\end{array}$ \\
\hline
\end{tabular}

tvalues were expressed in terms of Mean \pm SEM (SEM denoted; Standard Error of Mean) and values in brackets showed minimum-maximum values.

Inter-group comparison revealed that solely in Group II there was statistical significance among mean RBC, Hb, HCT and PLT values before and after treatment $(p \leq 0.05)$

other than Snap 4 Dx tests. Dogs enrolled in the present study were analyzed on referral with a qualitative ELISA assay able to determine $A$. phagocytophilum antibody. The aforementioned Snap 4 Dx test platform determines antibodies directed against a synthetic $A$. phagocytophilum peptide of a major surface protein, namely p44/MSP2, with sensitivity and specificity of 99 and $100 \%$, respectively, in comparison to an immunoflourescent assay (1).

During acute infection with vector-borne organisms, involving Anaplasma species, clinical signs may be evident before the dog has a measureable antibody response (10). From another point of view clinical signs usually present 1 or 2 weeks following tick transmission of Anaplasmosis, indeed.

The vast majority of exposed dogs may not develop overt clinical disease. According to the acute nature of the latter infection, about $40 \%$ of clinically ill dogs possess antibodies directed against CGA at the time of referral $(11,12)$. Thus therapy applications might not solely based on antibody testing, even if the results are negative or positive (10).

In a recent prospective study in which the present author (K.U.) was also involved, doxycycline-chloroquine combination therapy was successfull against Canine Monocytic Ehrlichiosis (13). The results of the latter study suggested doxycycline+chloroquine combination as efficacous for releiving of clinical signs during Canine Monocytic Ehrlichiosis. In the doxycycline+chloroquine group all of the hematological parameters analyzed returned to physiological levels after pudo haber estado expuesto al agente etiológico en los meses anteriores, asociado a la eliminación de la infección por inmunidad (10). Los ensayos de reacción en cadena de polimerasa pueden ser utilizados para detectar secuencias de ADN específicas al organismo en la sangre en las etapas tempranas (11). Por lo tanto, los autores del presente estudio pueden sugerir que los perros seropositivos objeto del presente estudio pueden haber sido infectados semanas e incluso meses antes, acorde con la descripción anterior (9).

La principal falla de la presente investigación fue que los resultados de pruebas serológicas o moleculares distintas a las pruebas Snap 4 Dx no estuvieron disponibles en el momento del diagnóstico. Los perros que participaron en el presente estudio fueron analizados al ser remitidos con un ensayo ELISA cualitativo para determinar el anticuerpo de A. phagocytophilum. La plataforma de dicha prueba Snap 4 Dx determina los anticuerpos dirigidos contra un péptido sintético de $A$. phagocytophilum de una proteína de superficie principal, a saber p44/MSP2, con una sensibilidad y una especificidad de $99 \%$ y $100 \%$ respectivamente, en comparación con un ensayo inmunofluorescente (1).

Durante la infección aguda por organismos transmitidos por vectores, que involucran especies de Anaplasma, los signos clínicos pueden ser evidentes antes de que el perro tenga una respuesta de anticuerpos medible (10). Desde otro punto de vista, los signos clínicos normalmente se presentan 1 o 2 semanas después de la transmisión efectiva de la Anaplasmosis por garrapatas.

La gran mayoría de los perros expuestos pueden no desarrollar una enfermedad clínica manifiesta. Según lo aguda que sea la infección, cerca del $40 \%$ de los perros clínicamente enfermos poseen anticuerpos para contrarrestar a la AGC en el momento en que son remitidos $(11,12)$. Por lo tanto, las aplicaciones de la terapia pueden no estar basadas exclusivamente en las pruebas de anticuerpos, independientemente de si los resultados son negativos o positivos (10).

En un estudio prospectivo reciente en el que estuvo involucrado el presente autor (K.U.), una terapia combinada de doxicilina+-cloroquina tuvo éxito en combatir la Ehrlichiosis Monocítica Canina (13). Los resultados de dicho estudio sugieren que la combinación doxicilina+cloroquina es igual de eficaz para aliviar signos clínicos en la Ehrlichiosis Monocítica Canina. En el grupo doxicilina + cloroquina, todos los parámetros hematológicos analizados volvieron a los niveles fisiológicos después del tratamiento (13). Los autores (13) también afirmaron que el tratamiento con doxicilina probablemente podría aliviar los efectos sistémicos 
this treatment (13). The latter authors (13) also claimed that treatment with doxycycline may probably alleviate the systemic effects of the tumor necrosis factor alpha, a cytokine that plays a role in the pathogenesis of acute canine ehrlichiosis, by reducing or eliminating parasitemia load (14). Furthermore chloroquine may have possessed anti-inflammatory effects $(15,16)$ and may have helped lowering the proinflammatory cytokines, or may be of beneficial for reducing infection of microbiological agents (17).

In another study 18 clinically ill dogs diagnosed with CGA by use of Snap 4 Dx analyte and PCR application, were treated with doxycycline (5-8 mg/kg q $12 \mathrm{~h}$, for 14-30 days), which resulted in rapid resolution (the vast majority of the dogs treated showed recovery in 1-2 days) of clinical signs (10). A 7-year-old Labrador retriever with nonspecific clinical signs was diagnosed within CGA based on serological studies and PCR testing. The dog recovered after treatment with tetracycline $(750 \mathrm{mg}, \mathrm{PO}, \mathrm{q} 8 \mathrm{~h}$ for 14 d) (18). Another case from Czech Republic, an 11-months-old male Golden Retriever developed the acute onset of fever, lameness, inappetence, depression, ataxia and reluctance to move, was diagnosed within Anaplasma phagocytophilum infection. Oral doxycycline treatment for 14 days at adosage of $10 \mathrm{mg} /$ $\mathrm{kg} \mathrm{q} 12 \mathrm{~h}$ resulted within resolution of clinical symptoms in six days (19).

Chloroquine is a 4-aminoquinoline derivative possessing antimalarial activity for treatment and prevention $(20,21)$. It has advantages due to its safety and effective usage, with a reasonable price of economic significance $(20,21)$. Regarding its 60 days half-live, chemoprophylactic effect occurs during the elimination phase $(21,22)$. Chloroquine might be safe therapy option for anti-inflammatory diseases. Although in the present study immunological parameters were not analyzed, chloroquine might have helped for a faster clinical remission in conjunction with doxycycline, as reported previously (13). However regarding hematological parameters evaluated, there was no statistical difference among groups treated solely with doxycycline or doxycycline + chloroquine. It should not be unwise to draw conclusion that larger dog population infected with CGA must be involved in studies evaluating chloroquine or similar antiprotozoer/antiinflammatory drug trials in an attempt to better understanding adjuvant therapy. Regardless of the interpretation, it must be kept in mind that Anaplasmosis may affect each dog differently, so alternative del factor alfa de necrosis tumoral, citoquina que juega un papel en la patogénesis de la ehrlichiosis canina aguda al reducir o eliminar la carga de parasitemia (14). Además, la cloroquina puede haber tenido efectos antiinflamatorios $(15,16)$ y puede haber contribuido a reducir las citoquinas proinflamatorias, o podría ser beneficiosa para reducir la infección por agentes microbiológicos (17).

En otro estudio, 18 perros clínicamente enfermos fueron diagnosticados con AGC utilizando la aplicación de analito en Snap 4 Dx y reacción en cadena de la polimerasa, y fueron tratados con doxicilina (5-8 mg/kg q $12 \mathrm{~h}$, durante 14-30 días) dando como resultado una rápida resolución (la gran mayoría de los perros que recibieron el tratamiento mostraron una rápida resolución (entre 1 y 2 días) de los signos clínicos (10). Un Labrador Retriever con signos no específicos fue diagnosticado con AGC con base en estudios serológicos y pruebas de reacción en cadena de la polimerasa. El perro se recuperó después del tratamiento con tetraciclina (750 mg, PO, q8h durante 14 d) (18). En otro caso ocurrido en la República Checa, un Golden Retriever varón de 11 años desarrolló un inicio agudo de fiebre, cojera, falta de apetito, depresión, ataxia y reticencia a moverse, por lo cual fue diagnosticado con infección de Anaplasma phagocytophilum. Se le administró un tratamiento oral de doxicilina durante 14 días con una dosis de $10 \mathrm{mg} / \mathrm{kg} \mathrm{q} 12 \mathrm{~h}$ que dio como resultado la resolución de los síntomas clínicos al cabo de seis días (19).

La cloroquina es un derivado de 4-aminoquinolina que posee una actividad antimalárica para el tratamiento y la prevención $(20,21)$. Presenta ventajas debido a que es segura y tiene un uso efectivo, a un precio razonable económicamente significativo $(20,21)$. Con respecto a su semivida de 60 días, el efecto quimoprofiláctico ocurre durante la fase de eliminación $(21,22)$. La cloroquina puede ser la opción terapéutica segura para enfermedades antiinflamatorias. Aunque en el presente estudio no se analizaron los parámetros inmunológicos, la cloroquina puede haber contribuido a una remisión clínica más rápida en conjunto con la doxicilina, de acuerdo a lo reportado previamente (13). Sin embargo, en cuanto a los parámetros hematológicos evaluados, no hubo una diferencia estadística entre los grupos tratados únicamente con doxicilina y los tratados con doxicilina+ cloroquina. No sería insensato llegar a la conclusión de que se debe involucrar a una población mayor de perros infectados con AGC en estudios que evalúen la cloroquina o en estudios farmacéuticos antiprotozarios/antiinflamatorios en aras de una mejor comprensión de la terapia adyuvante. Independientemente de la interpretación, se debe tener en cuenta que la Anaplasmosis puede 
therapy protocols, as shown in this study, might aid clinicians for dissolving related clinical signs resulting in better animal welfare. afectar a cada perro de forma distinta, por lo tanto los protocolos de terapia alternativos, según lo demuestra este estudio, pueden ayudar a los médicos a disolver los signos clínicos relacionados dando como resultado un mejor bienestar animal.

\section{REFERENCES}

1. Carrade D, Foley J, Sullivan M, Foley CW, Sykes JE. Spatial distribution of seroprevalence for Anaplasma phagocytophilum, Borrelia burgdorferi, Ehrlichia canis, and Dirofilaria immitis in dogs in Washington, Oregon, and California. Vet Clin Pathol 2011; 40(3):293-302.

2. Dumler JS, Barbet AF, Bekker CP. Reorganization of genera in the families Rickettsiaceae and Anaplasmataceae in the order Rickettsiales: unification of some species of Ehrlichia with Anaplasma, Cowdria with Ehrlichia and Ehrlichia with Neorickettsia, descriptions of six new species combinations and designation of Ehrlichia equi and 'HGE agent' as subjective synonyms of Ehrlichia phagocytophila. Int J Syst Evol Microbiol 2001;5:2145-2165.

3. Bakken JS, Dumler S. Human granulocytic anaplasmosis. Infect Dis Clin N Am 2008; 22:443-448.

4. Bakken JS, Dumler JS. Clinical diagnosis and treatment of human granulocytotropic anaplasmosis. Ann N Y Acad Sci 2006; 1078:236-247.

5. Bjöersdorff A. Ehrlichiosis and anaplasmosis, Part II: Anaplasma phagocytophilum comb. nov. infection. . Arthropod borne Infectious Diseases of the Dog and Cat. Manson Publishing, London; 2005.

6. Courtney JW, Kostelnik LM, Zeidner NS, Massung RF. Multiplex real time PCR for detection of Anaplasma phagocytophilum and Borrelia burgdorferi. J Clin Microbiol 2004; 42:3164-3168.

7. Carrade DD, Foley JE, Borjesson DL, Sykes JE. Canine granulocytic anaplasmosis: a review. J Vet Intern Med 2009; 23(6):1129-1141.

8. Bjoersdorff A. Canine granulocytic ehrlichiosis due to Anaplasma phagocytophila. Guide to Major Vector-borne Diseases of Pets, Merial, France. 2002.

9. McQuiston $\mathrm{JH}, \mathrm{McCall} \mathrm{CL}$, Nicholson WL. Ehrlichiosis and related infections. J Am Vet Med Assoc 2003; 223(12):1750-1756.
10. Eberts MD, Vissotto de Paiva Diniz PP, Beall MJ, Stillman BA, Chandrashekar $\mathrm{R}$, Breitschwerdt EB.Typical and atypical manifestations of Anaplasma phagocytophilum infection in dogs. J Am Anim Hosp Assoc 2011; 47(6):86-94.

11. Kohn B, Silaghi C, Galkea D, Arndt G, Pfister K. Infections with Anaplasma phagocytophilum in dogs in Germany. Res Vet Sci 2011; 91:71-76.

12. Granick JL, Armstrong PJ, Bender JB. Anaplasma phagocytophilum infection in dogs: 34 cases (2000-2007). J Am Vet Med Assoc 2009; 234(12):1559-65.

13. Aysul N, Ural K, Cetinkaya H, Kuşkucu M, Toros $\mathrm{G}$, Eren $\mathrm{H}$, Durum C. Doxycycline-Chloroquine Combination for the Treatment of Canine Monocytic Ehrlichiosis. Acta Sci Vet 2012; 40(2):1031.

14. Faria JL, Munhoz TD, João CF, VargasHernández G, André MR, Pereira WA, Machado RZ, Tinucci Costa M. Ehrlichia canis (Jaboticabal strain) induces the expression of TNF-a in leukocytes and splenocytes of experimentally infected dogs. Braz J Vet Parasitol 2011; 20(1):71-74.

15. Park J, Kwon D, Choi C, Oh JW, Benveniste EN. Chloroquine induces activation of nuclear factor kappa B and subsequent expression of proinflammatory cytokines by human astroglial cells. J Neurochem 2003; 84(6):1266-1274.

16. Weber S, Levitz SM. Chloroquine antagonizes the proinflammatory cytokine response to oppurtunistic fungi by alkalizing the fungal phagolysosome. J Infect Dis 2001; 183(6):935-942.

17. Wozniacka A, Lesiak A, Narbutt J, McCauliff DP, Sysa-Jedrzejowska A. Chloroquine treatment influences proinflammatory cytokine levels in systemic lupus erythematosus patients. Lupus 2006; 15(5):268-275. 
18. Lester SJ, Breitschwerdt EB, Hegarty BC. Anaplasma phagocytophilum infection (granulocytic anaplasmosis) in a dog from Vancouver Island. Can Vet J 2005; 46(9):825-827.

19. O Melter, I Stehlik, H Kinska, I Volfova, V Ticha, D Hulinska. Infection with Anaplasma phagocytophilum in a young dog: a case report Vet Med 2007; 52(5):207-212.

20. AlKadi HO. Antimalarial drug toxicity: a review. Chemotherapy 2007; 53(6):385-391.
21. Petersen I, Eastman R, Lanzer M. Drugresistant malaria: molecular mechanisms and implications for public health. FEBS Lett 2011; 585(11):1551-1562.

22. Stepniewska K, White NJ. Pharmacokinetic determinants of the window of selection for antimalarial drug resistance. Antimicrob Agents Chemother 2008; 52:1589-1596. 